\title{
Arctiin induces an UVB protective effect in human dermal fibroblast cells through microRNA expression changes
}

\author{
GHANG TAI LEE ${ }^{1 *}$, HWA JUN CHA ${ }^{2 *}$, KWANG SIK LEE $^{1}$, KUN KOOK LEE $^{1}$, JIN TAE HONG $^{3}$, \\ KYU JOONG AHN ${ }^{4}$, IN-SOOK AN ${ }^{2}$, SUNGKWAN AN ${ }^{2}$ and SEUNGHEE BAE ${ }^{2}$ \\ ${ }^{1}$ Songpa R\&D Center, Coreana Cosmetics Co., Ltd., Cheonan, Chungcheongnam-do 330-833; ${ }^{2}$ Molecular-Targeted \\ Drug Research Center and Korea Institute for Skin and Clinical Sciences, Konkuk University, Seoul 143-701; \\ ${ }^{3}$ College of Pharmacy and Medical Research Center, Chungbuk National University, Cheongju, \\ Chungcheongbuk-do 361-763; ${ }^{4}$ Department of Dermatology, Konkuk University \\ School of Medicine, Seoul 143-701, Republic of Korea
}

Received September 11, 2013; Accepted December 19, 2013

DOI: $10.3892 / \mathrm{ijmm} .2014 .1616$

\begin{abstract}
Ultraviolet (UV) radiation induces severe alterations in the molecular and cellular components of normal human dermal fibroblast (NHDF) cells by disrupting many intracellular transduction cascades. Although UV responses have been well documented at the genome and proteome levels, UV protective effects have not been elucidated at these levels. The aim of the present study was to demonstrate that arctiin, a phytochemical isolated from the plant Arctium lappa, induced a protective effect against UVB radiation by changing microRNA (miRNA) expression profiles. Using flow cytometry, and water-soluble tetrazolium salt (WST-1)-based cell viability, wound healing, and DNA repair assays we showed that pretreatment with arctiin prior to UVB irradiation reduced UVB-induced apoptosis, cell migration defects, and DNA damage in NHDF cells. It was also found that arctiin-induced UVB protection is associated with altered miRNA expression profiles. Bioinformatic analysis revealed that the deregulated miRNAs were functionally involved in mitogen-activated protein kinase (MAPK) signaling and cancer signaling pathways. The results suggest that arctiin acts as a UVB protective agent by altering specific miRNA expression in NHDF cells.
\end{abstract}

\section{Introduction}

Skin aging occurs in an age-dependent (internal aging) and environment-dependent (external aging) manner (1). Photoaging

Correspondence to: Dr Seunghee Bae, Molecular-Targeted Drug Research Center and Korea Institute for Skin and Clinical Sciences, Konkuk University, 120 Neungdong-ro, Gwangjin-gu, Seoul 143-701, Republic of Korea

E-mail:sbae@konkuk.ac.kr

${ }^{*}$ Contributed equally

Key words: arctiin, dermal fibroblast, ultraviolet B, microRNA, protection is a main component of extrinsic aging and is an important etiology of several skin diseases, such as photodermatoses, actinic keratosis and skin cancer (2). Photoaged skin exhibits severe alterations in the cellular component and extracellular matrix with atrophy of elastin, its microfibillar component fibrillin and interstitial collagens, major structural proteins of the dermis connective tissue (3). Ultraviolet (UV) radiation is a significant source of photoaging and although most UV radiation is blocked by the ozone layer of the stratosphere, there is increased awareness of the interactions between ozone depletion and climate change (global warming) that may have an impact on human exposure to terrestrial UV (4).

The effects of UV on human skin at the physiologic and molecular biologic levels have been previously investigated. UV radiation generates reactive oxygen species and DNA damage that induce cell cycle arrest and apoptosis (2). In addition, UV radiation transcriptionally induces the expression of matrix metalloproteinases (MMPs) and elastases, which affect cell migration and wrinkle formation through the degradation of collagen and elastin in dermal fibroblast cells (5). Mitogen-activated protein kinases (MAPKs) and nuclear factor- $\kappa \mathrm{B}(\mathrm{NF}-\kappa \mathrm{B})$ are post-translationally activated by UV through phosphorylation and their signaling pathways constitute a major molecular defense against UV radiation (6). Recently, gene expression profiling by microarray-based analysis revealed that many genes are regulated by UV-mediated signaling pathways $(7,8)$. Although there is extensive transcriptomic and proteomic analysis of UV signaling in skin cells, post-transcriptional responses to UV radiation, such as microRNAs, have not been widely studied.

MicroRNAs (miRNAs) are short RNA sequences ranging from 16 to 35 nucleotides that can directly interact with target mRNAs via complementary base pairing at a specific target site (9). The interaction between miRNA and target mRNA inhibits translation of the target, and results of previous studies suggested that $30 \%$ of all mRNAs in humans may be post-transcriptionally regulated by miRNAs $(9,10)$. In human dermal fibroblast cells, microarray-based studies have previously documented the molecular mechanisms underlying photodamage and skin carcinogenesis by UVB and UVA, 
and several miRNAs have been identified to be involved in this process $(11,12)$. In addition, miRNA-based studies have demonstrated that miR-34c-5p and miR-22 are UVB-response miRNAs and that the elevated expression of these miRNAs is capable of regulating UVB-mediated senescence and apoptosis in dermal fibroblasts $(13,14)$. Previously, we demonstrated that a titrated extract of Centella asiatica and epigallocatechin gallate (EGCG) have UVB protective effects in dermal fibroblast cells by altering miRNA expression profiles $(15,16)$. The abovementioned studies suggested that miRNA is likely a significant regulator of UVB-mediated cellular mechanisms and may also have protective functions in response to UVB.

Arctiin, a lignin compound derived from several plants including Arctium lappa, a herb used widely in traditional Chinese medicine for the treatment of the common cold (17). Findings of previous pharmacological studies revealed that arctiin possesses protective potential against lipopolysaccharide (LPS)-induced inflammation and 2-amino-1-methyl-6-phenylimidazo[4,5-b]pyridine (PhlP)-induced hepatocarcinogenesis and exerts an ameliorative effect on glomerulonephritis in Sprague-Dawley rat models (18-20). However, its protective effect in skin cells has not been investigated. To the best of our knowledge, this is the first study to demonstrate that arctiin exerts a protective effect against $U V B$ radiation in normal human dermal fibroblast (NHDF) cells by changing miRNA expression profiles.

\section{Materials and methods}

Cell culture and chemical treatment. NHDF cells (Lonza, Basel, Switzerland) were maintained in Dulbecco's modified Eagle's medium (DMEM) (Gibco-BRL, Invitrogen Life Technologies, Gaithersburg, MD, USA) containing 10\% fetal bovine serum (FBS; Sigma-Aldrich, St. Louis, MO, USA). Arctiin was purchased from Sigma-Aldrich and dissolved in DMSO. To evaluate the cytotoxicity and UVB protective effects of arctiin, NHDF cells were seeded in 96-well and $60-\mathrm{mm}$ culture plates at a density of $4 \times 10^{4}$ cells/well and $7 \times 10^{5}$ cells/plate, respectively.

UVB irradiation. UVB irradiation of NHDF cells was performed as described previously (15). Briefly, NHDF cells were seeded in 96-well and 60-mm plates and cultured in growth media overnight. When the cells reached $\sim 70 \%$ confluence they were pretreated with various doses of arctiin at different time points and then exposed to UVB irradiation. An irradiation dose of $100 \mathrm{~mJ} / \mathrm{cm}^{2}$ was used throughout the study. Prior to UVB irradiation the medium was replaced with phosphate-buffered saline (PBS) and the cells were exposed to UVB without the culture plate cover. Subsequent to UV exposure, the PBS was immediately replaced with growth medium containing DMSO or arctiin.

Cell viability assay. The cytotoxicity and UVB protective effects of arctiin on NHDF cells were investigated using a water-soluble tetrazolium salt (WST-1) assay (EZ-Cytox cell viability assay kit; Itsbio, Seoul, Korea). At the end of the experiments, $1 / 10$ volume of WST-1 solution was added to the cells and incubated at $37^{\circ} \mathrm{C}$ for $0.5 \mathrm{~h}$. Cell viability was determined by measuring the absorbance at $450 \mathrm{~nm}$ using an iMark microplate reader (Bio-Rad, Hercules, CA, USA). Results are presented as the means \pm standard deviation (SD) of three independent experiments. $\mathrm{P}<0.05$ as determined by Student's t-test was considered significant.

Analysis of cell cycle by flow cytometry. To distinguish cells in different phases of the cell cycle, UVB-irradiated NHDF cells pretreated with or without arctiin were fixed by the addition of cold $70 \%$ ethanol and stained with a fluorescent dye, propidium iodide (PI) (Sigma-Aldrich). The PI fluorescence intensity was detected using a FACSCalibur flow cytometer (BD Biosciences, San Jose, CA, USA). The mean PI fluorescence intensity was obtained from 10,000 cells using the FL2-H channel.

Wound healing assay. NHDF cells were seeded in 60-mm culture plates and grown overnight. When the cells reached $\sim 90 \%$ confluence they were pretreated with $10 \mu \mathrm{M}$ acrtiin for $6 \mathrm{~h}$ and a wound was formed by scraping the cells with a $20-\mu \mathrm{l}$ pipette tip and washing with PBS. After wound formation the cells were exposed to UVB irradiation and cultured in growth media containing DMSO or arctiin for $24 \mathrm{~h}$. Migration of the wounded cells was evaluated 0 and $24 \mathrm{~h}$ after wounding by recording photographic images using a phase-contrast Olympus CKX41 microscope (Olympus, Tokyo, Japan).

Luciferase-based DNA repair assay. To determine the effect of arctiin on DNA repair, the pGL3 luciferase reporter vector (Promega, Madison, WI, USA) was damaged with 2,000 J/m² UVC radiation as described previously (21). Control or damaged pGL3 vector was co-transfected into NHDF cells with pSV- $\beta$-galactosidase plasmid (as a transfection control) using Lipofectamine 2000 reagent (Invitrogen Life Technologies, Carlsbad, CA, USA). After $24 \mathrm{~h}$ the transfected cells were lysed using Passive Lysis buffer (Promega), luciferin was added, and the luciferase activity of each cell lysate was analyzed using a Veritas Luminometer (Turner Designs, Sunnyvale, CA, USA). The results were normalized to $\beta$-galactosidase activity and presented as percentages of the control with SD. Results shown are the averages of three independent experiments.

Total RNA purification. Total RNAs were purified using TRIzol reagent (Invitrogen Life Tecnologies) according to the manufacturer's instructions. The RNA integrity, concentration and purity were estimated using a Bioanalyzer 2100 (Agilent Technologies Inc., Santa Clara, CA, USA) and MaestroNano (Maestrogen, Las Vegas, NV, USA), respectively (15). RNA samples that showed A260/280 and A260/A230 values >1.8, and an RNA integrity number (RIN) $>8.0$ were subjected to microRNA-based microarray.

Microarray analysis of miRNA expression. Microarray analysis was performed using SurePrint G3 Human V16 miRNA 8x60K (Agilent Technologies Inc.), according to a previously described protocol (15). Briefly, 100 ng total RNA was labeled with cyanine 3-pCp and hybridized to the probes on the microarray. The microarray slide was scanned and data derived from the image were analyzed using GeneSpring GX software version 11.5 (Agilent Technologies Inc.). The raw data were filtered using FLAG and t-tests, and applied to the fold-change 


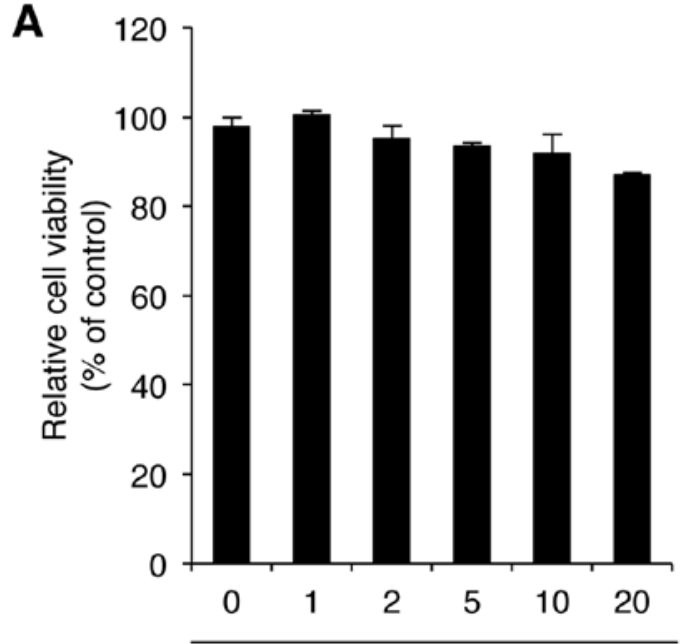

$\operatorname{Arctiin}(\mu \mathrm{M})$

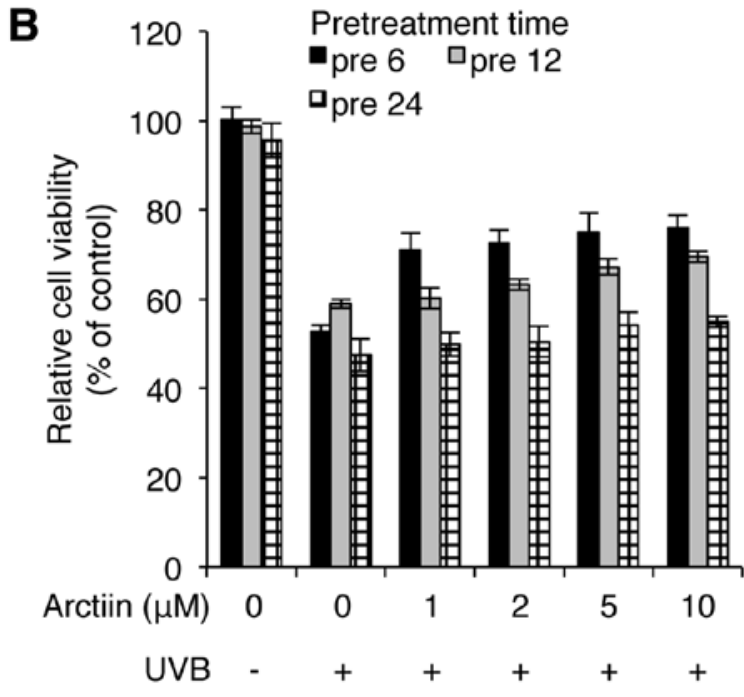

Figure 1. Ultraviolet (UV)B-mediated loss of normal human dermal fibroblast (NHDF) cell viability was decreased by pretreatment with arctiin. (A) Analysis of arctiin-mediated cytotoxicity in NHDF cells. Cells were seeded in 96-well culture dishes and treated with various doses of arctiin. After 24 h a water-soluble tetrazolium salt (WST-1)-based cytotoxicity assay was performed. Each bar represents mean \pm SD from three independent experiments. (B) Time- and dosedependence of the arctiin-mediated UVB protective effect on NHDF cells. Cells were pretreated with different doses of arctiin for various time points prior to UVB irradiation. Cell viabilities were determined using the WTS-1 assay. The graph represents mean \pm SD of relative cell viability from triplicate experiments.

analysis. Significant miRNAs were determined using the fluorescence ratio between two samples, and miRNAs exhibiting a $>2$-fold increase or decrease in expression were selected for subsequent bioinformatic analysis.

Bioinformatic analysis of deregulated miRNAs. To investigate the biological significance of the differentially expressed miRNAs, we first predicted putative target genes of the miRNAs using the DIANA-microT bioinformatic tool (http:// diana.imis.athena-innovation.gr/DianaTools/index.php? $r=$ microT_CDS/index) (22). The prediction of target genes was limited by setting a 0.8 -threshold in the program. The putative target genes of each miRNA were then analyzed for biological function using the Kyoto Encyclopedia of Genes and Genomes (KEGG) pathways and Database for Annotation, Visualization and Interrogate Discovery (DAVID) (http:// david.abcc.ncifcrf.gov/home.jsp) Bioinformatics Resources version 6.7 according to the developer's protocol (23). For example, the predicted 593 target genes of hsa-miR-1290 were uploaded into the DAVID web server and analyzed using the 'functional annotation tool' of DAVID. The 'KEGG pathway' category was then processed by setting a Ease score of 0.5. Involved KEGG pathways exhibiting a value of $>2 \%$ (percentage of involved target genes/total target genes) were selected.

\section{Results}

Arctiin promotes $U V B$ protection in NHDF cells. To determine whether arctiin is involved in the protection against UVB irradiation in NHDF cells, we first investigated the cytotoxic concentration range of arctiin. Treatment with 1-10 $\mu \mathrm{M}$ arctiin resulted in a $<10 \%$ decrease in cell viability whereas treatment with $20 \mu \mathrm{M}$ arctiin exhibited a higher cytotoxicity in NHDF cells (Fig. 1A). We also determined the UVB protection effect of arctiin by pretreating cells with arctiin at different concentrations $(1,2,5$ and $10 \mu \mathrm{M})$ and at different time points $(6,12$ and $24 \mathrm{~h})$ prior to UVB $\left(100 \mathrm{~mJ} / \mathrm{cm}^{2}\right)$ irradiation. Pretreatment with $10 \mu \mathrm{M}$ arctiin for $6 \mathrm{~h}$ showed the lowest decrease in viability after UVB irradiation, suggesting that this dose of arctiin has a photoprotective effect on UVB radiation in NHDF cells (Fig. 1B). Based on these results, pretreatment with $10 \mu \mathrm{M}$ arctiin for $6 \mathrm{~h}$ was used throughout the study.

Arctiin rescues $U V B$-induced apoptosis in NHDF cells. Treatment of NHDF cells with high doses of UVB irradiation leads to cell cycle arrest and apoptosis (24). To determine whether arctiin pretreatment affected the UVB-mediated physiological defects its effect on cell cycle distribution were analyzed using PI staining and flow cytometry. Treatment of NHDF cells with arctiin alone induced few changes in cell cycle distribution compared with the control cells (Fig. 2). UVB irradiation $\left(100 \mathrm{~mJ} / \mathrm{cm}^{2}\right)$ induced an increase in the number of sub-G1 cells (13.4\%), indicating that the dose of UVB radiation used in this study induced apoptosis in NHDF cells. Pre-treatment with arctiin prior to irradiation decreased the sub-G1 fraction (7.51\%), suggesting that arctiin protects against UVB-induced apoptosis in NHDF cells.

Arctiin rescues $U V B$-mediated migration defects in NHDF cells. Migration of NHDF cells is important for skin wound healing (25). To explore the possibility that arctiin rescues the UVB-induced migration defect in NHDF cells, we first analyzed whether arctiin induces NHDF migration. Arctiintreated cells showed a higher rate of migration than non-treated control cells after 24-h incubation, suggesting that arctiin accelerates the migration of NHDF cells (Fig. 3, upper two 


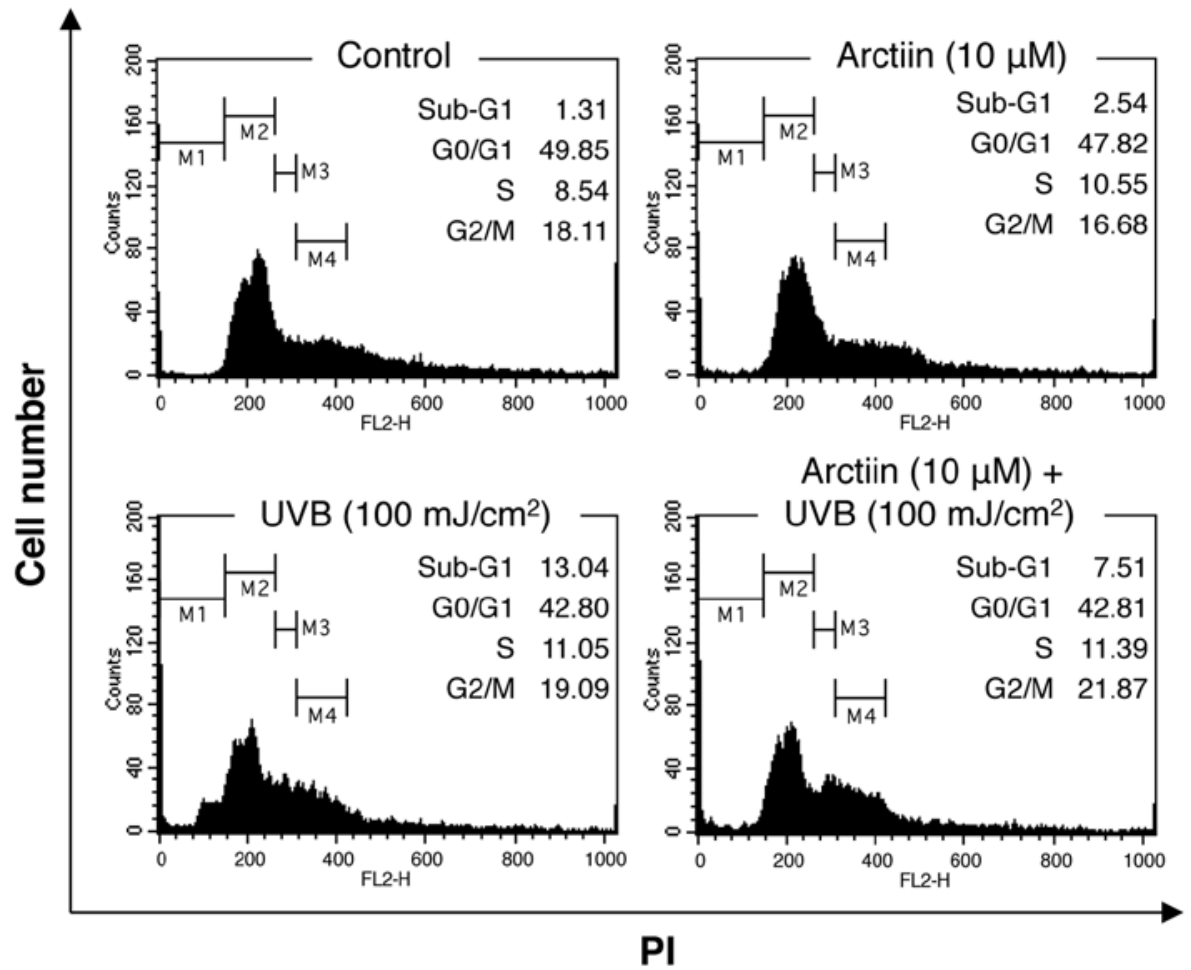

Figure 2. Ultraviolet (UV)B-induced apoptosis is rescued by arctiin treatment. Normal human dermal fibroblast (NHDF) cells were treated with DMSO or arctiin for $6 \mathrm{~h}$ prior to UVB irradiation. The cells were incubated for an additional $24 \mathrm{~h}$ and the level of apoptosis was measured by flow cytometry.

panels). We also confirmed that UVB irradiation markedly decreased the migration rate (Fig. 3, third panel) compared with the control. Arctiin pretreatment prior to UVB irradiation reduced the wound size compared with UVB irradiation alone. These results suggested that the UVB-mediated defect in wound healing was rescued by arctiin in NHDF cells.

Arctiin promotes UVB-mediated DNA damage repair. UV radiation induces cell death as a result of accumulation of DNA damage (26), thus we determined whether arctiin is involved in the repair of DNA damage induced by UV radiation in NHDF cells. pGL3 plasmid containing the luciferase gene was exposed to a $2,000 \mathrm{~J} / \mathrm{m}^{2}$ dose of UVC in vitro. Untreated or UVC-treated plasmids were cotransfected into NHDF cells with $\mathrm{pSV}$ - $\beta$-galactosidase plasmid as a transfection control. Following transfection, the cells were treated with $10 \mu \mathrm{M}$ arctiin for $24 \mathrm{~h}$ and the luciferase activities of cell lysates were measured as described in Materials and methods. The results showed that the luciferase activity decreased to $20.3 \%$ after UVC damage compared with the undamaged control (100\% luciferase activity). Pretreatment with arctiin did not affect luciferase activity of control pGL3 (Fig. 4), but rescued the decrease in luciferase activity induced by UVC irradiation to $43.8 \%$ (Fig. 4), suggesting that arctiin plays a protective role through the promotion of DNA damage repair in NHDF cells.

The UVB protection effect of arctiin is associated with changes in the expression of specific miRNAs in NHDF cells. $\mathrm{UV}$ radiation can alter the expression profiles of mRNA and microRNA (miRNA) in skin cells including NHDF cells (27). To explore the role of miRNAs in the arctiin-mediated UVB protection effect, we performed miRNA microarray analysis on NHDF cells. Total RNA from UVB-irradiated and arctiinpretreated/UVB-irradiated NHDF cells was hybridized against the SurePrint G3 Human v16 miRNA 8x60K microarray as described in Materials and methods. Numerous miRNAs showed significant differential expression, suggesting that arctiin induced expression changes in specific miRNAs to protect NHDF cells from UVB damage (Fig. 5A). Specifically, the expression level of 16 miRNAs was upregulated (Fig. 5A, left panel) and that of 10 miRNAs was downregulated (Fig. 5A, right panel) in the arctiin-mediated UVB protection system (Fig. 5B). Of the 26 miRNAs, hsa-miR-602 and -762 were upregulated 5.74- and 4.09-fold, respectively, and hsa-miR-765 and $-483-5 p$ were downregulated 11.86 - and 8.45 -fold, respectively. The complete list of differentially expressed miRNAs is provided in Table I.

Differentially regulated miRNAs may be involved in the regulation of key pathways involved in the UVB protection effect in NHDF cells. miRNA is an important regulator of cell proliferation, senescence and apoptosis through the modulation of target mRNA translation (28). Therefore, we investigated the biological significance of the deregulated miRNAs in the arctiin-mediated UVB protection effect. First, the predicted target genes of each miRNA were identified using the DIANA-microT-CDS (v5.0) bioinformatic tool as described in Materials and methods. To improve the accuracy of the target search, the threshold of the tool was fixed at 0.8. After the target search, information on the Ensembl transcript ID of target genes was collected and the ID lists of target genes were analyzed to identify their biological functions using DAVID bioinformatic resources. Biological significance was extracted from the large gene lists using 
Table I. miRNAs showing $>2$-fold expression change in NHDF cells pretreated with arctiin prior to UVB irradiation.

\begin{tabular}{|c|c|c|c|c|c|c|c|}
\hline miRNA & $\begin{array}{l}\text { Change } \\
\text { relative to } \\
\text { controls }\end{array}$ & $\begin{array}{l}\text { Direction of } \\
\text { regulation }\end{array}$ & Chr. & miRNA & $\begin{array}{l}\text { Change } \\
\text { relative to } \\
\text { controls }\end{array}$ & $\begin{array}{c}\text { Direction of } \\
\text { regulation }\end{array}$ & Chr. \\
\hline hsa-miR-1181 & 3.88 & Up & 19 & hsa-miR-762 & 4.09 & Up & 16 \\
\hline hsa-miR-150-3p & 3.17 & Up & 19 & hsa-miR-874 & 2.03 & Up & 5 \\
\hline hsa-miR-1915-3p & 2.12 & Up & 10 & hsa-miR-887 & 2.90 & Up & 5 \\
\hline hsa-miR-29b-1-5p & 2.72 & Up & 7 & hsa-miR-1246 & -3.81 & Down & 2 \\
\hline hsa-miR-3646 & 2.53 & Up & 20 & hsa-miR-1275 & -2.63 & Down & 6 \\
\hline hsa-miR-3663-3p & 2.51 & Up & 10 & hsa-miR-1290 & -2.37 & Down & 1 \\
\hline hsa-miR-3665 & 2.03 & Up & 13 & hsa-miR-3679-5p & -2.73 & Down & 2 \\
\hline hsa-miR-371a-5p & 2.64 & Up & 19 & hsa-miR-378b & -2.42 & Down & 3 \\
\hline hsa-miR-4327 & 2.95 & Up & 21 & hsa-miR-483-5p & -8.45 & Down & 11 \\
\hline hsa-miR-584-5p & 2.31 & Up & 5 & hsa-miR-501-5p & -2.25 & Down & $X$ \\
\hline hsa-miR-602 & 5.74 & Up & 9 & hsa-miR-575 & -2.08 & Down & 4 \\
\hline hsa-miR-629-3p & 2.95 & Up & 15 & hsa-miR-630 & -3.62 & Down & 15 \\
\hline hsa-miR-718 & 2.09 & Up & $X$ & hsa-miR-765 & -11.86 & Down & 1 \\
\hline
\end{tabular}

miRNAs, microRNAs; NHDF, normal human dermal fibroblast; UVB, ultraviolet B; Chr., chromosome.

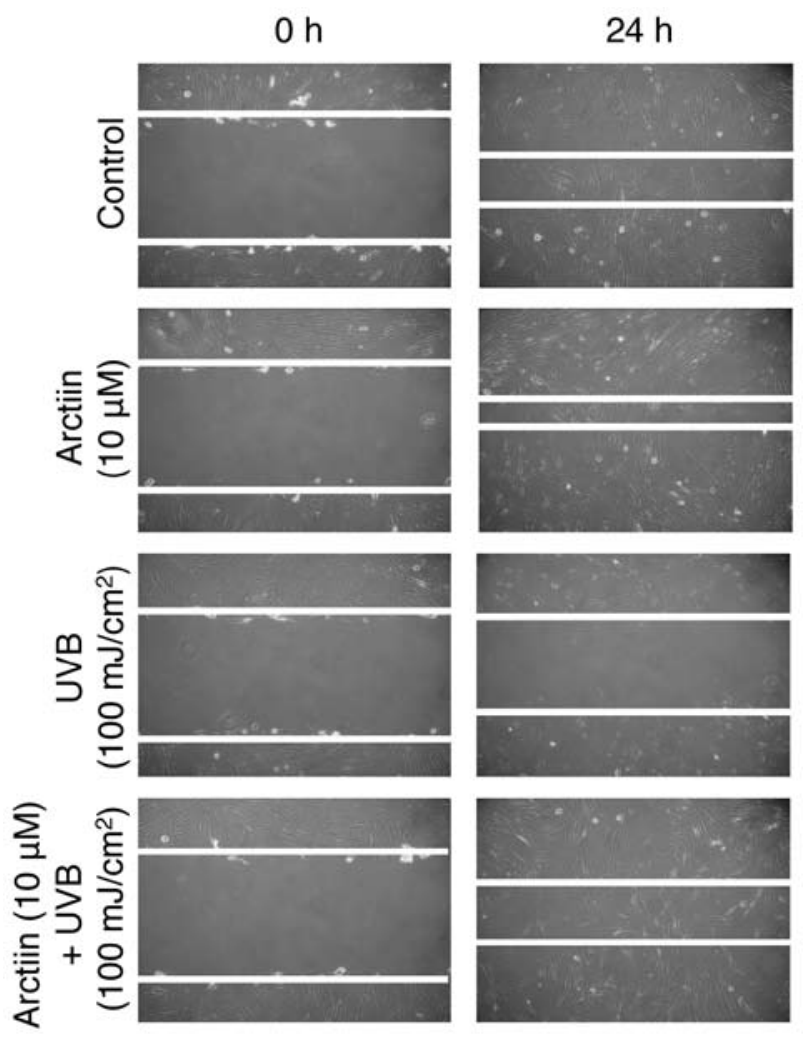

Figure 3. Ultraviolet (UV)B-induced defect in cell migration is decreased by arctiin treatment. Wounds were formed in confluent layers of untreated and arctiin-pretreated normal human dermal fibroblast (NHDF) cells prior to UVB irradiation. Phase-contrast images of the wounds were captured 0 and $24 \mathrm{~h}$ following irradiation.

one of the analysis tools available in the DAVID database or the KEGG pathway. To improve accuracy, the Ease score, which is a modified Fisher's exact P-value, was fixed at 0.5 and meaningful KEGG pathways showing a value of $>2 \%$

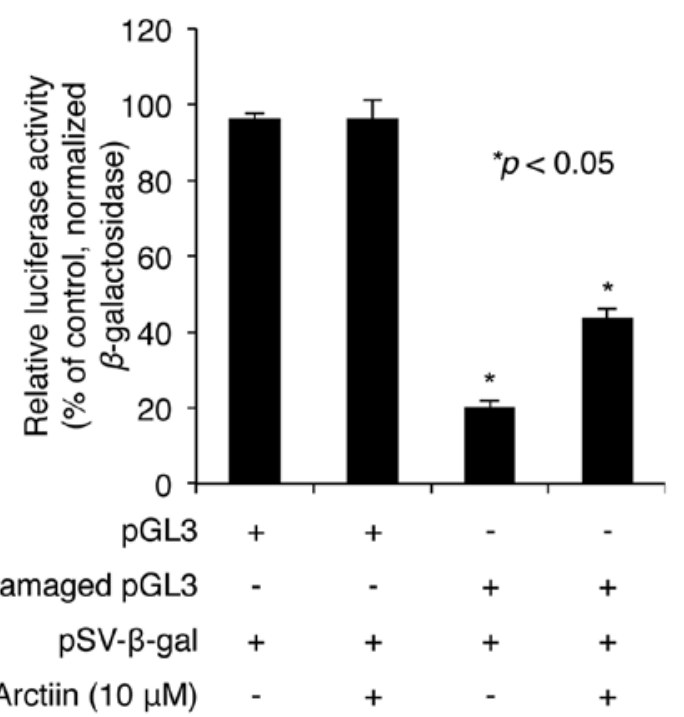

Figure 4. Arctiin induces repair of ultraviolet (UV)C-induced DNA damage. Prior to transfection, normal human dermal fibroblast (NHDF) cells were pretreated with arctiin for $6 \mathrm{~h}$. The pGL3 luciferase reporter plasmid was exposed to $2,000 \mathrm{~J} / \mathrm{m}^{2} \mathrm{UVC}$ and co-transfected with $\mathrm{pSV}-\beta$-galactose plasmid as a transfection control. Undamaged pGL3 plasmid was used as a control for UV damage. Cells were grown in arctiin-containing media for a further $24 \mathrm{~h}$ and luciferase activities in whole cell lysates were measured. Data are the average of three replicate experiments with mean \pm SD. ${ }^{*} \mathrm{P}<0.05$ compared with control.

(percentage of involved target genes/total target genes) were selected. The results suggested these miRNAs were present in the signaling pathways of cell cycle, cell proliferation, cancer, ubiquitin-mediated proteolysis, insulin, focal adhesion, MAPK, Wnt and ErbB (Tables II and III). In particular, almost all of the upregulated miRNAs were involved in MAPK, Wnt and cancer signaling pathways, whereas the downregulated miRNAs were mainly involved in MAPK, ErbB, focal 
Table II. Main functions of upregulated miRNAs predicted by bioinformatics analysis.

\begin{tabular}{|c|c|c|c|c|c|}
\hline miRNA & Total targets & KEGG pathway & $\begin{array}{c}\text { Target } \\
\text { count in } \\
\text { pathway }\end{array}$ & $\%^{\mathrm{a}}$ & P-value \\
\hline \multirow[t]{4}{*}{ hsa-miR-602 } & 302 & MAPK signaling pathway & 7 & 2.3 & $2.20 \mathrm{E}-01$ \\
\hline & & Insulin signaling pathway & 6 & 2 & $5.30 \mathrm{E}-02$ \\
\hline & & Alzheimer's disease & 6 & 2 & $1.00 \mathrm{E}-01$ \\
\hline & & Calcium signaling pathway & 6 & 2 & $1.30 \mathrm{E}-01$ \\
\hline \multirow[t]{5}{*}{ hsa-miR-762 } & 534 & Axon guidance & 16 & 3 & $6.60 \mathrm{E}-07$ \\
\hline & & MAPK signaling pathway & 16 & 3 & $2.90 \mathrm{E}-03$ \\
\hline & & Pathways in cancer & 15 & 2.8 & $3.70 \mathrm{E}-02$ \\
\hline & & Wnt signaling pathway & 13 & 2.4 & $4.00 \mathrm{E}-04$ \\
\hline & & Regulation of actin cytoskeleton & 11 & 2.1 & $4.50 \mathrm{E}-02$ \\
\hline hsa-miR-1181 & 2 & - & - & - & - \\
\hline \multirow[t]{4}{*}{ hsa-miR-150-3p } & 184 & Wnt signaling pathway & 5 & 2.7 & $6.00 \mathrm{E}-02$ \\
\hline & & Neurotrophin signaling pathway & 4 & 2.2 & $1.20 \mathrm{E}-01$ \\
\hline & & Ubiquitin-mediated proteolysis & 4 & 2.2 & $1.50 \mathrm{E}-01$ \\
\hline & & MAPK signaling pathway & 4 & 2.2 & 4.90E-01 \\
\hline hsa-miR-629-3p & 445 & Pathways in cancer & 10 & 2.3 & $2.10 \mathrm{E}-01$ \\
\hline \multirow[t]{4}{*}{ hsa-miR-4327 } & 112 & MAPK signaling pathway & 4 & 3.6 & $1.20 \mathrm{E}-01$ \\
\hline & & Pathways in cancer & 4 & 3.6 & $1.80 \mathrm{E}-01$ \\
\hline & & Melanoma & 3 & 2.7 & $4.00 \mathrm{E}-02$ \\
\hline & & Calcium signaling pathway & 3 & 2.7 & $1.90 \mathrm{E}-01$ \\
\hline hsa-miR-887 & 10 & - & - & - & - \\
\hline hsa-miR-29b-1-5p & 265 & - & - & - & - \\
\hline \multirow[t]{2}{*}{ hsa-miR-371a-5p } & 351 & Spliceosome & 8 & 2.3 & $4.20 \mathrm{E}-03$ \\
\hline & & Wnt signaling pathway & 7 & 2 & $3.60 \mathrm{E}-02$ \\
\hline hsa-miR-3646 & 569 & - & - & - & - \\
\hline \multirow[t]{5}{*}{ hsa-miR-3663-3p } & 305 & MAPK signaling pathway & 12 & 3.9 & $5.90 \mathrm{E}-03$ \\
\hline & & Pathways in cancer & 11 & 3.6 & $5.50 \mathrm{E}-02$ \\
\hline & & Neurotrophin signaling pathway & 7 & 2.3 & $2.00 \mathrm{E}-02$ \\
\hline & & Focal adhesion & 7 & 2.3 & $1.30 \mathrm{E}-01$ \\
\hline & & Cytokine-cytokine receptor interaction & 7 & 2.3 & $3.00 \mathrm{E}-01$ \\
\hline \multirow[t]{2}{*}{ hsa-miR-584-5p } & 288 & MAPK signaling pathway & 8 & 2.8 & $9.70 \mathrm{E}-02$ \\
\hline & & Pathways in cancer & 8 & 2.8 & $2.10 \mathrm{E}-01$ \\
\hline \multirow[t]{2}{*}{ hsa-miR-1915-3p } & 351 & Wnt signaling pathway & 8 & 2.3 & $5.60 \mathrm{E}-03$ \\
\hline & & Pathways in cancer & 7 & 2 & $3.30 \mathrm{E}-01$ \\
\hline hsa-miR-718 & 18 & - & - & - & - \\
\hline \multirow[t]{2}{*}{ hsa-miR-874 } & 176 & B- and T-cell receptor signaling pathway & 4 & 2.3 & $2.20 \mathrm{E}-02$ \\
\hline & & MAPK signaling pathway & 4 & 2.3 & $3.70 \mathrm{E}-01$ \\
\hline \multirow[t]{3}{*}{ hsa-miR-3665 } & 195 & Neurotrophin signaling pathway & 4 & 2.1 & $1.10 \mathrm{E}-01$ \\
\hline & & Insulin signaling pathway & 4 & 2.1 & $1.30 \mathrm{E}-01$ \\
\hline & & MAPK signaling pathway & 4 & 2.1 & 4.70E-01 \\
\hline
\end{tabular}

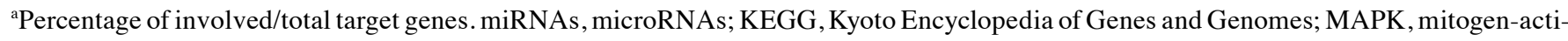
vated protein kinase.

adhesion, cell cycle and cancer signaling pathways. Of note, the MAPK signaling pathway, which involves MAPKK, p38, JNK and ERK1/2, was one of the most significant pathways identified for up- and downregulated miRNAs.

\section{Discussion}

Although arctiin, a major lignin of Arctium lappa, has been reported to have various biological functions including 
Table III. Main functions of downregulated miRNAs predicted by bioinformatics analysis.

\begin{tabular}{|c|c|c|c|c|c|}
\hline miRNA & Total targets & KEGG pathway & $\begin{array}{c}\text { Target } \\
\text { count in } \\
\text { pathway }\end{array}$ & $\%^{\mathrm{a}}$ & $\mathrm{P}$-value \\
\hline hsa-miR-765 & 548 & Cytokine-cytokine receptor interaction & 11 & 2 & $2.00 \mathrm{E}-01$ \\
\hline hsa-miR-483-5p & 32 & Focal adhesion & 2 & 6.2 & $1.50 \mathrm{E}-01$ \\
\hline hsa-miR-1246 & 290 & Neurotrophin signaling pathway & 7 & 2.4 & $3.20 \mathrm{E}-03$ \\
\hline hsa-miR-630 & 54 & Alanine and glutamate metabolism & 2 & 3.7 & $4.80 \mathrm{E}-02$ \\
\hline \multirow[t]{4}{*}{ hsa-miR-3679-5p } & 238 & Calcium signaling pathway & 7 & 2.9 & $1.80 \mathrm{E}-02$ \\
\hline & & ErbB signaling pathway & 5 & 2.1 & $2.00 \mathrm{E}-02$ \\
\hline & & Insulin signaling pathway & 5 & 2.1 & $7.80 \mathrm{E}-02$ \\
\hline & & MAPK signaling pathway & 5 & 2.1 & 4.00E-01 \\
\hline \multirow[t]{3}{*}{ hsa-miR-1275 } & 268 & Tight junction & 8 & 3 & $1.10 \mathrm{E}-03$ \\
\hline & & Wnt signaling pathway & 6 & 2.2 & $3.60 \mathrm{E}-02$ \\
\hline & & Endocytosis & 6 & 2.2 & $7.20 \mathrm{E}-02$ \\
\hline \multirow[t]{2}{*}{ hsa-miR-378b } & 162 & Pathways in cancer & 8 & 4.9 & $3.60 \mathrm{E}-02$ \\
\hline & & Cell cycle & 4 & 2.5 & $1.20 \mathrm{E}-01$ \\
\hline \multirow[t]{4}{*}{ hsa-miR-1290 } & 593 & Pathways in cancer & 17 & 2.9 & $4.00 \mathrm{E}-02$ \\
\hline & & Focal adhesion & 14 & 2.4 & $7.90 \mathrm{E}-03$ \\
\hline & & Insulin signaling pathway & 13 & 2.2 & $7.60 \mathrm{E}-04$ \\
\hline & & MAPK signaling pathway & 12 & 2 & $1.90 \mathrm{E}-01$ \\
\hline \multirow[t]{3}{*}{ hsa-miR-501-5p } & 301 & Ubiquitin-mediated proteolysis & 9 & 3 & $7.60 \mathrm{E}-04$ \\
\hline & & MAPK signaling pathway & 8 & 2.7 & $9.20 \mathrm{E}-02$ \\
\hline & & Regulation of actin cytoskeleton & 6 & 2 & $2.00 \mathrm{E}-01$ \\
\hline \multirow[t]{5}{*}{ hsa-miR-575 } & 241 & MAPK signaling pathway & 8 & 3.3 & $7.70 \mathrm{E}-02$ \\
\hline & & Non-small cell lung cancer & 6 & 2.5 & $8.60 \mathrm{E}-04$ \\
\hline & & Prostate cancer & 6 & 2.5 & $7.70 \mathrm{E}-03$ \\
\hline & & Melanoma & 5 & 2.1 & $1.70 \mathrm{E}-02$ \\
\hline & & Cell cycle & 5 & 2.1 & $9.60 \mathrm{E}-02$ \\
\hline
\end{tabular}

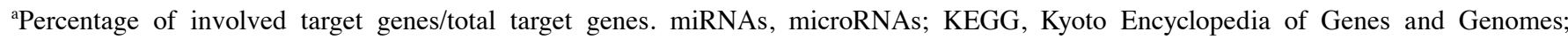
MAPK, mitogen-activated protein kinase.

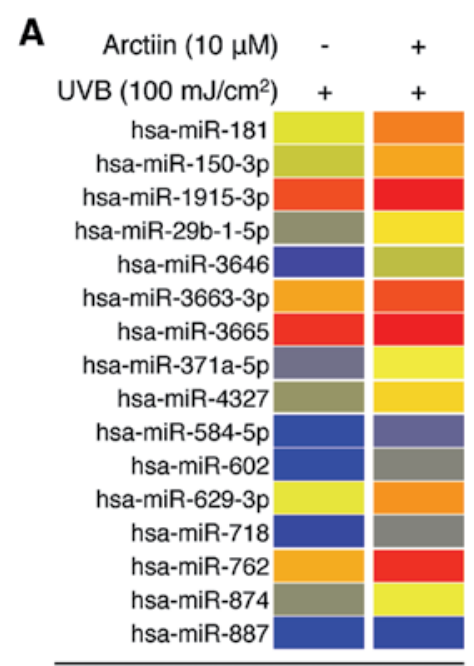

Upregulated miRNAs

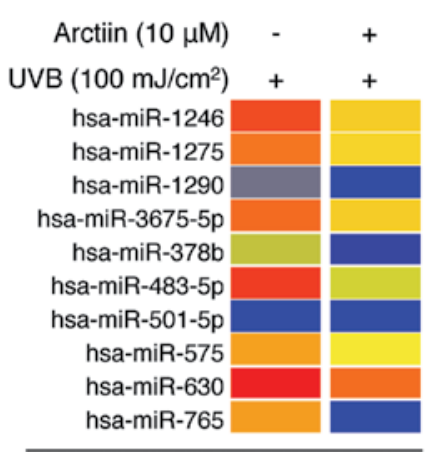

Downregulated miRNAs

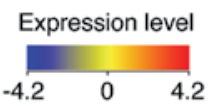

B

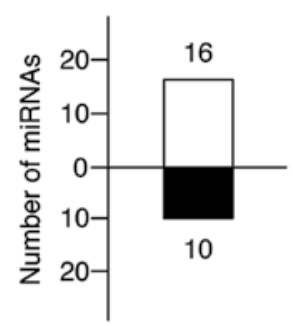

Upregulated

Downregulated

Figure 5. Arctiin-mediated ultraviolet (UV)B protection is associated with changes in microRNA (miRNA) expression profiles. (A) Heat-map of deregulated miRNAs that have 2-fold higher (upregulated) or lower (downregulated) cy3 fluorescence in cells treated with arctiin before irradiation compared with DMSO-treated control cells. (B) Graphshowing the number of deregulated miRNAs. 
anti-microbial, anti-inflammatory and anticancer properties $(19,29,30)$, the antiproliferative functions of arctiin, which are documented in PC3 prostate cancer cells, HepG2 hepatocarcinoma cells and $\mathrm{HaCaT}$ keratinocytes (30-32), remain controversial. Matsuzaki et al (30) showed that relatively high doses of arctiin $(25-250 \mu \mathrm{M})$ decreased the number of viable cells and increased the fraction of cells in G1 phase to $10 \%$, but did not induce apoptosis. In addition, Huang et al (31) showed that arctiin preferentially induced cell detachment, but did not exert anti-proliferative or cytotoxic effects in PC3 cells. Furthermore, our results show that relatively low doses $(1,2,5$ and $10 \mu \mathrm{M})$ of arctiin exhibited little cytotoxicity but $20 \mu \mathrm{M}$ arctiin induced a $14 \%$ decrease in cell viability. These results suggest that, although arctiin induces cell detachment in specific cell types, the antiproliferative effect may be evident only at high doses, and low concentrations of arctiin may exert other biological activities that have not yet been identified.

To the best of our knowledge we have demonstrated, for the first time, that a low concentration of arctiin protects NHDF cells against UVB damage. Results of the WST-1-based cell viability assay revealed that pretreatment with $10 \mu \mathrm{M}$ arctiin for $6 \mathrm{~h}$ conferred maximum UVB protection. We also confirmed that arctiin inhibits UVB-induced apoptosis, cell migration defect and DNA damage. Notably, longer pretreatment (12 and $24 \mathrm{~h}$ ) with arctiin did not enhance the protection effect over a 6-h treatment. Viability of cells pretreated for $24 \mathrm{~h}$ with various doses of arctiin was comparable to that of UVB-irradiated cells. It has been reported $(30,31)$ that arctiin-mediated inhibition of cell growth is dependent on treatment time. Cells treated with or without arctiin for $<24 \mathrm{~h}$ showed a similar growth rate, but cells treated with arctiin for $>24 \mathrm{~h}$ showed decreased growth rates. Similarly, treatment with 3 and $10 \mu \mathrm{M}$ arctiin for short periods of time exert no cytotoxic effects, whereas treatment for $>30 \mathrm{~h}$ resulted in a significantly decreased cell growth rate. Therefore, the arctiin-mediated UVB protection effect is dependent on treatment time.

A recent study focusing on the possible role and function of miRNAs in UVB-mediated skin diseases such as skin aging and cancer provided a novel viewpoint on the pathogenesis of UV radiation-related diseases in human skin (27). miRNAs have been shown to be important in a large number of specific skin physiological processes, including keratinocyte differentiation, melanogenesis, development of skin stem cells and dermal fibroblast senescence (14,33-36). Microarray analyses have revealed that the expression profiles of miRNAs are altered in photodamage and skin carcinogenesis induced by UV radiation, and numerous miRNAs are involved in this process $(11,27,34)$. Moreover, changes in miRNA profiles may be directly related to the pathogenesis of photoaging and skin cancer. However, the UVB protective effect of miRNAs in NHDF cells has not been extensively investigated and little is known regarding the potential role of photodamage-regulated miRNAs in cell function, which may be an important factor in the progression of diseases related to UVB radiation. In the present study, the miRNA-based microarray analysis revealed that the arctiin-mediated UVB protection effect involved deregulation of 26 miRNAs in NHDF cells, of which 16 were upregulated and 10 were downregulated. Of the 26 miRNAs, the expression of 8 miRNAs altered $>3$-fold. In their study,
Zhou et al (14) showed that UVB radiation, not only changed the expression profiles of miRNAs, but that specific miRNAs are major inducers of UVB-mediated senescence. These deregulated miRNAs potentially have an important effect on biological pathways essential for protection against UVB in skin cells.

It has been reported that $>30 \%$ of protein-coding genes are post-transcriptionally regulated by miRNAs, where each miRNA is able to target 200 transcripts and a single mRNA may also be targeted by $>1$ miRNA $(9,10)$. To determine the possible biological effects of the miRNAs that are deregulated during arctiin-mediated UVB protection in NHDF cells, we first predicted the target genes of the miRNAs using bioinformatic tools with a high threshold, and then performed a functional gene-annotation enrichment analysis using the KEGG pathway database. The results showed that the deregulated miRNAs were primarily associated with the cancer, MAPK, Wnt, insulin signaling and neurotrophin signaling pathways. Of these, the MAPK pathway was one of the most significantly involved pathways affected by miRNAs that were up- or downregulated in arctiin-treated UVB-irradiated cells. The upregulated miRNAs, including miR-602, -762, -150-3p, $-4327,-584-5 p,-874$ and -3665 were all strongly predicted to affect target genes involved in the MAPK pathway. Several of the downregulated miRNAs, including miR-3679-5p, -1290 and -575 , were also predicted to be involved in the MAPK pathway. This signaling cascade is involved in a number of cell functions, including proliferation, differentiation and apoptosis. In a recent study, UV was found to activate MAPKs such as p38 MAPK, JNK and ERK1/2, which play important roles in the UV response in skin cells (6). Notably, activation of p38 MAPK and JNK is critically involved in UV-mediated proapoptotic and antiapoptotic responses (6), whereas activated ERK1/2 has been reported only in UV-mediated proapoptotic signal transduction (6). Therefore, ERK1/2 activation may be specifically regulated by the miRNAs that are upregulated by arctiin in NHDF cells. The study also reported that UV-mediated activation of those MAPKs is not post-transcriptional, but is induced by post-translational modifications such as phosphorylation (6). Therefore, our finding that the target genes of up- and downregulated miRNAs are significantly involved in the MAPK pathway raises the possibility that upstream or downstream proteins of MAPKs are post-transcriptionally regulated by the deregulated miRNAs and may contribute to the arctiin-mediated UVB protection effect in NHDF cells.

The role of miRNA-602 in cell functions has previously been studied in hepatitis B virus-mediated hepatocellular carcinoma (37). The data revealed that miR-602 regulated the tumor suppressor gene RASS1FA, which affects several cell functions including proliferation, migration, senescence and apoptosis (38). Loss of RASSF1 expression is associated with the status of K-ras, which is an effector molecule of MAPK signal transduction (39). Considering that miR-602 was the most highly upregulated miRNA in our arctiin-mediated UVB protection system, it is likely that the upregulated expression of miR-602 contributes at least in part to UVB protection in NHDF cells. miR-765 and -483-5p were the most highly downregulated miRNAs identified in the present study. The cell function of those miRNAs has not been investigated; 
however, the aberrant expression of miR-765 and -483-5p may also be involved in UVB protection.

In summary, to the best of our knowledge, we have demonstrated for the first time that a low dose of arctiin has UVB protective activity in NHDF cells. We also confirmed that arctiin inhibits the UVB-mediated cell growth defect, apoptosis, cell migration defect and DNA damage in these cells. Furthermore, arctiin treatment induces expression changes in specific miRNA profiles, and some of the deregulated miRNAs have predicted roles in the regulation of MAPK and cell growth signal pathways. Although future studies should be performed to validate the deregulated miRNAs, results of this study have provided novel information on miRNA-mediated UVB protection in NHDF cells.

\section{Acknowledgements}

We would like to thank all other members of Coreana Cosmetics Co., Ltd. for their support. This study was supported by the KU Research Professor Program of Konkuk University and grant from the Ministry of Science, ICT and Future Planning (grant no. 20110028646) of the Republic of Korea.

\section{References}

1. Sjerobabski-Masnec I and Situm M: Skin aging. Acta Clin Croat 49: 515-518, 2010.

2. Wang B: Photoaging: a review of current concepts of pathogenesis. J Cutan Med Surg 15 (Suppl 1): S374-S377, 2011.

3. Wlaschek M, Tantcheva-Poór I, Naderi L, et al: Solar UV irradiation and dermal photoaging. J Photochem Photobiol B 63: 41-51, 2001.

4. Diffey B: Climate change, ozone depletion and the impact on ultraviolet exposure of human skin. Phys Med Biol 49: R1-R11, 2004.

5. Philips N, Auler S, Hugo R and Gonzalez S: Beneficial regulation of matrix metalloproteinases for skin health. Enzyme Res 2011 427285, 2011.

6. Muthusamy V and Piva TJ: The UV response of the skin: a review of the MAPK, NFkappaB and TNFalpha signal transduction pathways. Arch Dermatol Res 302: 5-17, 2010.

7. Travers JB, Edenberg HJ, Zhang Q, et al: Augmentation of UVB radiation-mediated early gene expression by the epidermal platelet-activating factor receptor. J Invest Dermatol 128: 455-460, 2008.

8. Casati P and Walbot V: Gene expression profiling in response to ultraviolet radiation in maize genotypes with varying flavonoid content. Plant Physiol 132: 1739-1754, 2003.

9. Bartel DP: MicroRNAs: target recognition and regulatory functions. Cell 136: 215-233, 2009.

10. Griffiths-Jones S, Saini HK, van Dongen S and Enright AJ: miRBase: tools for microRNA genomics. Nucleic Acids Res 36: D154-D158, 2008.

11. Li W, Zhou BR, Hua LJ, Guo Z and Luo D: Differential miRNA profile on photoaged primary human fibroblasts irradiated with ultraviolet A. Tumour Biol: Jul 7, 2013 (Epub ahead of print).

12. Lu C, Ding ZH and Zhou MJ: Mechanisms of ultraviolet B irradiation-induced injuries in $16 \mathrm{HBE}$ cells. Nan Fang Yi Ke Da Xue Xue Bao 31: 57-60, 2011 (In Chinese).

13. Tan G, Shi Y and Wu ZH: MicroRNA-22 promotes cell survival upon UV radiation by repressing PTEN. Biochem Biophys Res Commun 417: 546-551, 2012.

14. Zhou BR, Guo XF, Zhang JA, et al: Elevated miR-34c-5p mediates dermal fibroblast senescence by ultraviolet irradiation. Int J Biol Sci 9: 743-752, 2013.

15. An IS, An S, Kang SM, et al: Titrated extract of Centella asiatica provides a UVB protective effect by altering microRNA expression profiles in human dermal fibroblasts. Int $\mathbf{J}$ Mol Med 30: 1194-1202, 2012.
16. An IS, An S, Park S, Lee SN and Bae S: Involvement of microRNAs in epigallocatechin gallate-mediated UVB protection in human dermal fibroblasts. Oncol Rep 29: 253-259, 2013.

17. Sun WJ, Sha ZF and Gao H: Determination of arctiin and arctigenin in Fructus Arctii by reverse-phase HPLC. Yao Xue Xue Bao 27: 549-551, 1992.

18. Hirose M, Yamaguchi T, Lin C, et al: Effects of arctiin on $\mathrm{PhIP}$-induced mammary, colon and pancreatic carcinogenesis in female Sprague-Dawley rats and MeIQx-induced hepatocarcinogenesis in male F344 rats. Cancer Lett 155: 79-88, 2000.

19. Lee S, Shin S, Kim H, et al: Anti-inflammatory function of arctiin by inhibiting COX-2 expression via NF- $\mathrm{B}$ pathways. J Inflamm (Lond) 8: 16, 2011.

20. Wu JG, Wu JZ, Sun LN, et al: Ameliorative effects of arctiin from Arctium lappa on experimental glomerulonephritis in rats. Phytomedicine 16: 1033-1041, 2009.

21. Cui X, Zhang J, Du R, et al: HSF4 is involved in DNA damage repair through regulation of Rad51. Biochim Biophys Acta 1822: 1308-1315, 2012

22. Maragkakis M, Reczko M, Simossis VA, et al: DIANA-microT web server: elucidating microRNA functions through target prediction. Nucleic Acids Res 37: W273-W276, 2009.

23. Huang da W, Sherman BT and Lempicki RA: Systematic and integrative analysis of large gene lists using DAVID bioinformatics resources. Nat Protoc 4: 44-57, 2009.

24. Lee $\mathrm{CH}$, Wu SB, Hong $\mathrm{CH}$, Yu HS and Wei YH: Molecular mechanisms of UV-induced apoptosis and its effects on skin residential cells: the implication in UV-based phototherapy. Int J Mol Sci 14: 6414-6435, 2013.

25. Singer AJ and Clark RA: Cutaneous wound healing. N Engl J Med 341: 738-746, 1999.

26. Sinha RP and Häder DP: UV-induced DNA damage and repair: a review. Photochem Photobiol Sci 1: 225-236, 2002.

27. Syed DN, Khan MI, Shabbir M and Mukhtar H: MicroRNAs in skin response to UV radiation. Curr Drug Targets 14: 1128-1134, 2013.

28. Subramanyam D and Blelloch R: From microRNAs to targets: pathway discovery in cell fate transitions. Curr Opin Genet Dev 21: 498-503, 2011.

29. Hayashi K, Narutaki K, Nagaoka Y, Hayashi T and Uesato S: Therapeutic effect of arctiin and arctigenin in immunocompetent and immunocompromised mice infected with influenza A virus. Biol Pharm Bull 33: 1199-1205, 2010.

30. Matsuzaki Y, Koyama M, Hitomi T, et al: Arctiin induces cell growth inhibition through the down-regulation of cyclin D1 expression. Oncol Rep 19: 721-727, 2008.

31. Huang DM, Guh JH, Chueh SC and Teng CM: Modulation of anti-adhesion molecule MUC-1 is associated with arctiininduced growth inhibition in PC-3 cells. Prostate 59: 260-267, 2004.

32. Moritani S, Nomura M, Takeda Y and Miyamoto K: Cytotoxic components of bardanae fructus (goboshi). Biol Pharm Bull 19: 1515-1517, 1996.

33. Yi R and Fuchs E: MicroRNA-mediated control in the skin. Cell Death Differ 17: 229-235, 2010.

34. Zhou BR, Xu Y, Permatasari F, et al: Characterization of the miRNA profile in UVB-irradiated normal human keratinocytes. Exp Dermatol 21: 317-319, 2012.

35. Bemis LT, Chen R, Amato CM, et al: MicroRNA-137 targets microphthalmia-associated transcription factor in melanoma cell lines. Cancer Res 68: 1362-1368, 2008.

36. Hildebrand J, Rütze M, Walz N, et al: A comprehensive analysis of microRNA expression during human keratinocyte differentiation in vitro and in vivo. J Invest Dermatol 131: 20-29, 2011.

37. Yang L, Ma Z, Wang D, Zhao W, Chen L and Wang G: MicroRNA-602 regulating tumor suppressive gene RASSF1A is overexpressed in hepatitis B virus-infected liver and hepatocellular carcinoma. Cancer Biol Ther 9: 803-808, 2010.

38. Fernandes MS, Carneiro F, Oliveira C and Seruca R: Colorectal cancer and RASSF family--a special emphasis on RASSF1A. Int J Cancer 132: 251-258, 2013.

39. Cao D, Chen Y, Tang Y, et al: Loss of RASSF1A expression in colorectal cancer and its association with K-ras status. Biomed Res Int 2013: 976765, 2013. 